\title{
ON THE DERIVATIVE OF A POLYNOMIAL AND CHEBYSHEV APPROXIMATION ${ }^{1}$
}

\section{T. S. MOTZKIN AND J. L. WALSH}

Introduction. The location of the zeros of the derivative of a polynomial has been much studied, as has the location of the zeros of the Chebyshev polynomial. In $\$ 1$ of the present note we set forth in a direct and elementary manner the equivalence of these two problems in a suitably specialized situation. This conclusion is mentioned (for integral $\lambda_{i}$ ) with an indication of the proof by Fekete and von Neumann [4],2 and the conclusion is related to a much deeper investigation due to Fekete [3]. We obtain ( $\$ 9)$ some new results on zeros of approximating polynomials and $(\$ \S 2,3,8)$ on the argument of the deviation. In $\$ 10$ we consider approximation by an arbitrary linear family. Throughout the paper we study primarily approximation on a set of $n$ points by a polynomial of degree $n-2$. In $\$ \$ 4-7$ weight functions with infinities and Chebyshev rational functions are introduced.

1. Determination of special Chebyshev polynomials with weight function. If $E$ is a closed bounded point set of the $z$-plane on which the weight function $\mu(z)$ is positive and continuous, the Chebyshev polynomial $T_{m}(z)$ of degree $m$ for $E$ with weight function $\mu(z)$ is defined as that polynomial of the form

$$
z^{m}+A_{1} z^{m-1}+\cdots+A_{m}
$$

for which the norm

$$
\max \left[\mu(z)\left|T_{m}(z)\right|, z \text { on } E\right]
$$

is least. It can be shown that $T_{m}(z)$ exists and is unique. In the special case about to be considered the existence and uniqueness of $T_{m}(z)$ will be established.

THEOREM. Let the point set $E$ consist of the distinct points $z_{1}, z_{2}, \cdots$, $z_{n}(n>1)$ and let $u$ set $\omega(z) \equiv\left(z-z_{1}\right)\left(z-z_{2}\right) \cdots\left(z-z_{n}\right)$. Then the unique Chebyshev polynomial $T_{n-1}(z)$ of degree $n-1$ for $E$ with the weight function $\mu\left(z_{i}\right) \equiv \mu_{i}=1 / \lambda_{i}\left|\omega^{\prime}\left(z_{i}\right)\right|$, where $\lambda_{i}>0, \sum_{1}^{n} \lambda_{i}=1$, is the 1952.

Presented to the Society, September 7, 1951; received by the editors January 25 ,

1 The preparation of this paper was sponsored (in part) by the Office of Scientific Research, USAF.

${ }^{2}$ Figures in brackets indicate the literature references at the end of this paper. 
polynomial

$$
T_{n-1}(z) \equiv \omega(z) \sum_{1}^{n} \frac{\lambda_{i}}{z-z_{i}} .
$$

Note that we consider here essentially the most general positive weight function on $E$, for multiplication of the weight function by a positive constant does not alter the Chebyshev polynomial.

We observe that $T_{n-1}(z)$ as defined by (2) is of form (1), and that from the definition of $\omega^{\prime}\left(z_{i}\right)$ as a limit we have $T_{n-1}\left(z_{i}\right)=\lambda_{i} \omega^{\prime}\left(z_{i}\right)$. The norm of $T_{n-1}(z)$ on $E$ is thus

$$
\max \left[\mu_{i}\left|T_{n-1}\left(z_{i}\right)\right|\right]=1 \text {. }
$$

The Lagrange interpolation formula represents (as may be verified directly) an arbitrary polynomial of degree ${ }^{3} n-1$ in terms of its values in the $n$ points $z_{i}$, and in the present case becomes

$$
T_{n-1}(z) \equiv \sum_{1}^{n} \frac{T_{n-1}\left(z_{i}\right)}{\omega^{\prime}\left(z_{i}\right)} \frac{\omega(z)}{z-z_{i}} \equiv \omega(z) \sum_{1}^{n} \frac{\lambda_{i}}{z-z_{i}},
$$

which is (2).

An arbitrary polynomial of the form $P(z) \equiv z^{n-1}+B_{1} z^{n-2}+\cdots$ $+B_{n-1}$ is expressed by Lagrange's formula

$$
P(z) \equiv \sum_{1}^{n} \frac{P\left(z_{i}\right)}{\omega^{\prime}\left(z_{i}\right)} \frac{\omega(z)}{z-z_{i}} ;
$$

inspection of the terms of highest degree in $z$ yields

$$
1=\sum_{1}^{n} \frac{P\left(z_{i}\right)}{\omega^{\prime}\left(z_{i}\right)} \text {. }
$$

The norm of $P(z)$ on $E$ is

$$
\max \left[\mu_{i}\left|P\left(z_{i}\right)\right|\right]=\max \left[\left|P\left(z_{i}\right) / \lambda_{i} \omega^{\prime}\left(z_{i}\right)\right|\right] .
$$

We contrast (3) with the equation $1=\sum_{1}^{n} \lambda_{i}$, where we have $\lambda_{i}>0$. Either for some value of $i$ we have $\left|P\left(z_{i}\right) / \omega^{\prime}\left(z_{i}\right)\right|>\lambda_{i}$, in which case the norm (4) of $P(z)$ is greater than unity, the norm of $T_{n-1}(z)$, or we have $\left|P\left(z_{i}\right) / \omega^{\prime}\left(z_{i}\right)\right|=\lambda_{i}$ for every $i$, in which case all the quotients $P\left(z_{i}\right) / \omega^{\prime}\left(z_{i}\right)$ are real and positive and we have $P\left(z_{i}\right) / \omega^{\prime}\left(z_{i}\right)$ $=\lambda_{i}$ for every $i$, whence $P(z) \equiv T_{n-1}(z)$. That is to say, we have shown that $T_{n-1}(z)$ as defined by (2) is the unique polynomial of form (1)

${ }^{3}$ As is customary in the study of approximation, we define a polynomial of degree $n-1$ as an arbitrary function of the form $A_{0} z^{n-1}+A_{1} z^{n-2}+\cdots+A_{n-1}$. 
of least norm on $E$; the theorem is established.

We note that $T_{n-1}(z)$ is $\omega(z)$ multiplied by the logarithmic derivative of

$$
\prod_{1}^{n}\left(z-z_{i}\right)^{\lambda_{i}}, \quad \sum_{1}^{n} \lambda_{i}=1,
$$

where of course the $\lambda_{i}$ need not be commensurable. Thus the study of the zeros of the derivative of (5) is identical with the study of the zeros of $T_{n-1}(z)$.

2. Properties of the arguments of the Chebyshev polynomial. The equation $T_{n-1}\left(z_{i}\right)=\lambda_{i} \omega^{\prime}\left(z_{i}\right)$ shows that for each $z_{i}$ the arguments of the $T_{n-1}\left(z_{i}\right)$ are fixed independently of the choice of the $\lambda_{k}$ :

$$
\arg \left[T_{n-1}\left(z_{i}\right)\right]=\arg \left[\omega^{\prime}\left(z_{i}\right)\right] .
$$

By a new application of Lagrange's formula we have a representation of the polynomial unity:

$$
1 \equiv \sum_{1}^{n} \frac{1}{\omega^{\prime}\left(z_{i}\right)} \frac{\omega(z)}{z-z_{i}} ;
$$

inspection of the terms of highest degree gives $(n>1)$

$$
0=\sum_{1}^{n} \frac{1}{\omega^{\prime}\left(z_{i}\right)},
$$

which we write in the form

$$
\sum_{1}^{n} \frac{\lambda_{i}}{\lambda_{i} \omega^{\prime}\left(z_{i}\right)}=0,
$$

and by taking conjugates write this also in the form

$$
\sum_{1}^{n} \lambda_{i} \omega^{\prime}\left(z_{i}\right) \frac{1}{\lambda_{i}\left|\omega^{\prime}\left(z_{i}\right)\right|^{2}}=0 .
$$

Equation (7) states that if the points $\lambda_{i} \omega^{\prime}\left(z_{i}\right)$ are interpreted in the $w$-plane, then the origin is the center of gravity of those points considered with suitably chosen positive weights, namely $1 / \lambda_{i}\left|\omega^{\prime}\left(z_{i}\right)\right|^{2}$. Consequently the origin lies in the smallest convex polygon containing (i.e., lies in the convex hull of) the points $\lambda_{2} \omega^{\prime}\left(z_{i}\right)=T_{n-1}\left(z_{i}\right)$, and lies interior to this polygon except when the polygon degenerates to a line segment; in the latter case the origin is an interior point of the segment. This conclusion is familiar in the case that all the points $z_{i}$ are real and each $\lambda_{i}$ equal to $1 / n$; here it follows from (2) that we 
have $T_{n-1}(z) \equiv \omega^{\prime}(z) / n$; the values of $\lambda_{i} \omega^{\prime}\left(z_{i}\right)=\omega^{\prime}\left(z_{i}\right) / n$ are alternately positive and negative if $z_{1}<z_{2}<\cdots<z_{n}$; the origin lies interior to the smallest line segment containing the points $\lambda_{i} \omega^{\prime}\left(z_{i}\right)$.

Equation (6) obviously shows that the origin lies in the convex hull of the points $1 / \lambda_{i} \omega^{\prime}\left(z_{i}\right)$.

3. Examples. It is of interest to add some examples where $\mu_{i}, \lambda_{i}$, and $\left|\omega^{\prime}\left(z_{i}\right)\right|$ are all independent of $i$; thus $\lambda_{i}=1 / n$, and $T_{n-1}(z) \equiv \omega^{\prime}(z) / n$. In each example $E$ is defined as the set of zeros of $\omega(z)$.

EXAMPLE 1. $\omega(z) \equiv z^{n}-1, T_{n-1}(z) \equiv \omega^{\prime}(z) / n \equiv z^{n-1}$.

EXAMPLE 2. $\omega(z) \equiv\left(z^{n}-\alpha\right)\left(z^{n}-\bar{\alpha}\right), \alpha \neq 0, T_{2 n-1}(z) \equiv \omega^{\prime}(z) / 2 n$.

EXAMPLE 3. $\omega(z) \equiv z\left(z^{n}-\alpha\right)\left(z^{n}-\bar{\alpha}\right),|\alpha|=1,|\alpha-\bar{\alpha}|=1 / n, T_{2 n}(z) \equiv$ $\omega^{\prime}(z) /(2 n+1)$.

4. Weight functions with infinities. Let now $E$ no longer necessarily consist of $n$ points. It is entirely permissible not to require that the given weight function $\mu(z)$ be continuous on the given set $E$; for instance $\mu(z)$ may be of the form

$$
\mu(z) \equiv \frac{\mu_{1}(z)}{\left|\left(z-\alpha_{1}\right) \cdots\left(z-\alpha_{k}\right)\right|},
$$

where $\mu_{1}(z)$ is positive and continuous on $E$ and the points $\alpha_{1}, \alpha_{2}, \cdots$, $\alpha_{k}$ not necessarily distinct belong to $E$. The norm of a polynomial $T_{m}(z)$ is as before $\max \left[\mu(z)\left|T_{m}(z)\right|, z\right.$ on $\left.E\right]$; even if $\alpha_{i}$ is an isolated point of $E$, the value $\mu\left(\alpha_{i}\right)\left|T_{m}\left(\alpha_{i}\right)\right|$ is to be interpreted as

$$
\lim _{z \rightarrow \alpha_{i}} \mu_{1}\left(\alpha_{i}\right)\left|T_{m}(z)\right| /\left|\left(z-\alpha_{1}\right) \cdots\left(z-\alpha_{k}\right)\right|
$$

provided the limit (finite or infinite) exists. For the Chebyshev polynomial $T_{m}(z)$ properly to exist, the norm of $T_{m}(z)$ is to be finite, we must have $m \geqq k$, and all the points $\alpha_{i}$ must be zeros of $T_{m}(z)$. If we write

$$
T_{m}(z) \equiv\left(z-\alpha_{1}\right)\left(z-\alpha_{2}\right) \cdots\left(z-\alpha_{k}\right) S_{m}(z),
$$

the norm of $T_{m}(z)$ is $\max \left[\mu_{1}(z)\left|S_{m}(z)\right|, z\right.$ on $\left.E\right]$, where $S_{m}(z) \equiv z^{m-k}$ $+A_{1} z^{m-k-1}+\cdots+A_{m-k}$ is of degree $m-k$; the problem of studying the Chebyshev polynomial $T_{m}(z)$ of degree $m(\geqq k)$ with weight function $\mu(z)$ is identical with the problem of studying the Chebyshev polynomial $S_{m}(z)$ of degree $m-k$ with weight function $\mu_{1}(z)$.

5. Chebyshev rational functions. The use of a weight function of form (8) is related to Chebyshev rational functions with prescribed poles. For simplicity let $E$ be a closed and bounded point set of the 
z-plane on which the weight function $\mu(z)$ is positive and continuous, and let the prescribed points $\alpha_{1}, \alpha_{2}, \cdots, \alpha_{k}$ be disjoint from $E$. The Chebyshev rational function $R_{m}(z)$ is the function of form

$$
R_{m}(z) \equiv \frac{z^{m}+B_{1} z^{m-1}+\cdots+B_{m}}{\left(z-\alpha_{1}\right)\left(z-\alpha_{2}\right) \cdots\left(z-\alpha_{k}\right)}
$$

of least norm. Here we have precisely the problem of the Chebyshev polynomial $T_{m}(z)$ on $E$ with the norm function

$$
\frac{\mu(z)}{\left|\left(z-\alpha_{1}\right)\left(z-\alpha_{2}\right) \cdots\left(z-\alpha_{k}\right)\right|} .
$$

It is of course imperative either to require that the numerator of $R_{m}(z)$ should be of the form indicated in (9) or in some other way to exclude as inadmissible the trivial function $R_{m}(z) \equiv 0$.

6. Fejer's theorem. We return to the general Chebyshev polynomial $T_{m}$ given by (1) for a set $E$ containing at least $m$ points. Beyond the case where all points of $E$ are collinear, the first geometric result on the zeros of $T_{m}(z)$ is due to Fejér [2]: All zeros of $T_{m}(z)$ lie in the convex hull of $E$. The proof is not difficult. Let the point $z_{0}$ lie exterior to $K$, the convex hull of $E$, and let $\beta$ be the point of $K$ nearest $z_{0}$. Then we have in each point $z$ of $E$

$$
\left|z-\left(z_{0}+\beta\right) / 2\right|<\left|z-z_{0}\right| \text {. }
$$

For any polynomial $Q(z) \equiv z^{m-1}+\cdots$, we therefore have at each point $z$ of $E$ with $Q(z) \neq 0$

$$
\left|\left[z-\left(z_{0}+\beta\right) / 2\right] Q(z)\right|<\left|\left(z-z_{0}\right) Q(z)\right| .
$$

Thus $\left(z-z_{0}\right) Q(z)$ cannot be a Chebyshev polynomial for $E$ with positive weight function.

As Fekete and von Neumann (loc. cit.) point out, by considering (5) there follows the theorem of Lucas, that the convex hull of the zeros of a polynomial contains the zeros of the derived polynomial.

7. Poles of extremal rational functions. The theorem of Fejér implies that the finite zeros of the C.r.f. $R_{m}(z)$ of form (9) lie in the convex hull of $E$. If we consider even a more general extremal problem for rational functions, where now the $\alpha_{i}$ are allowed to vary in suitable regions, it is clear that the C.r.f. for this more general problem is also the C.r.f. for the former problem where the $\alpha_{i}$ are suitably determined and considered fixed. It is still true that the finite zeros of $R_{m}(z)$ lie in the convex hull $K$ of $E$; moreover the reasoning used in 
connection with (10) now applies in reverse: if $\alpha_{i}$ exterior to $K$ is a pole of the C.r.f. and if $L$ is the half line through $\alpha_{i}$ from the nearest point of $K$, then $\alpha_{i}$ must be on $L$ as far away from $K$ as possible.

8. Approximation to an arbitrary function. The term Chebyshev polynomial is also used in a sense different from that defined in $\$ 1$. If $E$ is a closed bounded point set, if $\mu(z)$ is a weight function positive and continuous on $E$, and if $f(z)$ is continuous on $E$, then the Chebysher polynomial of best approximation $t_{m}(z)$ is the polynomial of degree $m$ such that the norm

$$
\max \left[\mu(z)\left|f(z)-t_{m}(z)\right|, z \text { on } E\right]
$$

is least. It can be shown that $t_{m}(z)$ exists and is unique; we shall prove this result in the special case which concerns us below.

Suppose that $E$ consists of precisely $n$ distinct points. Let $f_{1}(z)$ $\equiv B_{0} z^{n-1}+B_{1} z^{n-2}+\cdots+B_{n-1}$ be the unique polynomial of degree $n-1$ which coincides with $f(z)$ on $E$. In the case $m=n-2$, the norm (11) becomes

$$
\max \left[\mu(z)\left|f_{1}(z)-t_{n-2}(z)\right|, z \text { on } E\right] .
$$

If $B_{0}=0$, the polynomial $f_{1}(z)$ is itself a polynomial of degree $n-2$, admissible in the consideration of all approximating polynomials, whence $t_{n-2}(z) \equiv f_{1}(z)$. Henceforth we suppose $B_{0}=\sum_{1}^{n} f\left(z_{i}\right) / \omega^{\prime}\left(z_{i}\right) \neq 0$, so that (12) can be written

$$
\begin{aligned}
& \max \left[\left|B_{0}\right| \mu(z) \mid z^{n-1}+B_{1} z^{n-2} / B_{0}+\cdots\right. \\
&\left.+B_{n-1} / B_{0}-t_{n-2}(z) / B_{0} \mid, z \text { on } E\right]
\end{aligned}
$$

The problem of minimizing (13) is precisely the problem of determining the Chebyshev polynomial $T_{n-1}(z)$ for $E$ with weight function $\left|B_{0}\right| \mu(z)$, a problem that we have already discussed. In particular it follows that $t_{n-2}(z)$ exists and is unique. If we define the numbers

$$
\lambda_{i}=\frac{1}{\mu\left(z_{i}\right)\left|\omega^{\prime}\left(z_{i}\right)\right|} / \sum_{1}^{n} \frac{1}{\mu\left(z_{i}\right)\left|\omega^{\prime}\left(z_{i}\right)\right|},
$$

we have by (2)

$$
\frac{f_{1}(z)-t_{n-2}(z)}{B_{0}} \equiv T_{n-1}(z) \equiv \omega(z) \sum_{1}^{n} \frac{\lambda_{i}}{z-z_{i}},
$$

from which $t_{n-2}(z)$ can be determined; the second factor in this last member is the logarithmic derivative of (5).

The norm (12) can now be computed by setting $T_{n-1}\left(z_{i}\right)=\lambda_{i} \omega^{\prime}\left(z_{i}\right)$ : 


$$
\begin{aligned}
\mu\left(z_{i}\right)\left|f\left(z_{i}\right)-t_{n-2}\left(z_{i}\right)\right| & =\left|B_{0}\right| \mu\left(z_{i}\right)\left|T_{n-1}\left(z_{i}\right)\right| \\
& =\left|B_{0}\right| / \sum_{1}^{n} \frac{1}{\mu\left(z_{i}\right)\left|\omega^{\prime}\left(z_{i}\right)\right|},
\end{aligned}
$$

which is obviously independent of $i$. Again, as in $\$ 2$, the value of $\arg \left\{\left[f(z)-t_{n-2}(z)\right] / B_{0}\right\}=\arg \left[T_{n-1}(z)\right]=\arg \left[\omega^{\prime}(z)\right]$ in each point of $E$ is independent of the particular choice of $\mu(z)$ and of $f(z)$. The origin lies in the convex hull of the points $\left[f\left(z_{i}\right)-t_{n-2}\left(z_{i}\right)\right]$, and lies interior to this polygon except when the polygon degenerates to a line segment.

In the special examples given in $\$ 3$ the value of $\arg \{[f(z)$ $\left.\left.-t_{n-2}(z)\right] / B_{0}\right\}=\arg \left[\omega^{\prime}(z)\right]$ can be written down at once.

All zeros of the polynomial $f_{1}(z)-t_{n-2}(z)$ lie in the convex hull of $E$ (if $B_{0} \neq 0$ ).

Specific formulas for the norm (12) have been obtained by de la Vallée Poussin [1]. He also proves that a necessary and sufficient condition that given numbers $\delta_{i}$, all different from zero, be deviations of the approximating polynomial $t_{n-2}(z)$ from some function $f(z)$ in the $n$ points $z_{i}$ is that for no polynomial $p_{n-2}(z)$ of degree $n-2$ all the quotients $p_{n-2}\left(z_{i}\right) / \delta_{i}$ have positive real parts. Thus we may now state: $a$ necessary and sufficient condition that for given $\delta_{i}(\neq 0)$ no polynomial $p_{n-2}(z)$ of degree $n-2$ exists such that all the guotients $p_{n-2}\left(z_{i}\right) / \delta_{i}$ have positive real parts is $\arg \delta_{i}=\arg \omega^{\prime}\left(z_{i}\right)$. Hence if this condition is satisfied, the origin $O$ lies in the convex hulls of the points $p_{n-2}\left(z_{i}\right) / \delta_{i}$, and of those points $\delta_{i} / p_{n-2}\left(z_{i}\right)$ which are finite. This includes the result of $\$ 2$.

This characterization of the arguments of $\delta_{i}$ is an extension to the complex domain of the alternation of signs of the $\delta_{i}$ in the real domain; compare \$2. However, as de la Vallée Poussin indicates, there is an essential difference in that approximation on an arbitrary closed bounded set $E$ in the real domain by polynomials of degree $n-2$ is equivalent to approximation on a subset of $n$ points of $E$, whereas such approximation in the complex domain is equivalent to approximation on a subset of $E$ containing $m$ points, $n \leqq m \leqq 2 n-1$. In the present paper we consider only the case $m=n$; for other $m$ the arguments of the $\delta_{i}$ are no longer uniquely determined.

9. Location of zeros of approximating polynomials. We proceed to devote some attention to the zeros of the polynomials that occur in $\S 8$, with $B_{0} \neq 0$, using the notation of $\S 8$.

The function $f(z)$ considered on the set $E:\left(z_{1}, z_{2}, \cdots, z_{n}\right)$ is entirely arbitrary, so the polynomial $f_{1}(z)$ is an arbitrary polynomial of degree $n-1$ except for the restriction $B_{0} \neq 0$, and nothing can be de- 
duced concerning its zeros without further hypothesis. Likewise the polynomial $t_{n-2}(z)$ given by

$$
t_{n-2}(z) \equiv f_{1}(z)-B_{0} T_{n-1}(z)
$$

can be considered an arbitrary polynomial of degree $n-2$, provided $f(z)$ is arbitrary. However, under suitable conditions we can derive certain conclusions regarding these polynomials:

Let the zeros of the polynomial $f_{1}(z)$ lie in the closed interior (respectively exterior) $C_{1}$ of the circle $|z-\alpha|=r_{1}$, and let the zeros of $T_{n-1}(z)$ lie in the closed interior $C_{2}$ of the circle $|z-\beta|=r_{2}$ (for which it is sufficient that $E$ lie in $C_{2}$ ), where $C_{1}$ and $C_{2}$ are disjoint. Then all zeros of $t_{n-2}(z)$ in (15) lie in the $n-2$ closed regions

$$
\begin{aligned}
& \left|z-\frac{\alpha-\epsilon \beta}{1-\epsilon}\right| \leqq \frac{r_{1}+r_{2}}{|1-\epsilon|}, \\
& \left|z-\frac{\alpha-\epsilon \beta}{1-\epsilon}\right| \geqq \frac{r_{1}-r_{2}}{|1-\epsilon|},
\end{aligned}
$$

respectively, where $\epsilon$ takes all the values except unity of the $(n-1)$ st roots of unity. If the circles (16) are mutually exterior, they contain each one zero of $t_{n-2}(z)$.

Inequalities (16) follow [6] from equation (15), since the coefficient of $z^{n-1}$ in $f_{1}(z)$ is $B_{0}$, and inequalities (17) can be similarly proved.

In the special case in which (15) is used to define $T_{n-1}(z)$ itself, we set $f_{1}(z) \equiv z^{n-1}, B_{0}=1, \alpha=0, r_{1}=0$.

It is essential to assume that $C_{1}$ and $C_{2}$ are disjoint; otherwise we may have, e.g., $t_{n-2}(z) \equiv 0$.

All the circles (16) (resp. (17)) lie [7] in the closed exterior of the hyperbola (ellipse) $C$ whose foci are $\alpha$ and $\beta$, and whose transverse (major) axis is $r_{1}+r_{2}$ (resp. $r_{1}-r_{2}$ ); the centers of the circles are equidistant from $\alpha$ and $\beta$, and the circles are doubly tangent to $C .^{4}$

10. Approximation by functions of a linear family. We turn now to best approximation on the set $E:\left(z_{1}, z_{2}, \cdots, z_{n}\right), n>1$, to the function $f(z)$ by functions of the form $\sum_{1}^{n-1} c_{j} f_{j}(z)$, where the $f_{j}(z)$ are arbitrary functions defined on $E$ and the complex constants $c_{j}$ are to be determined so that

$$
\max \left[\left|f(z)-\sum_{1}^{n-1} c_{j} f_{j}(z)\right|, z \text { on } E\right]
$$

\footnotetext{
4 If $C$ is an ellipse, the double tangency may occur merely in the formal algebraic sense.
} 
is least. We suppose the functions $f_{j}(z)$ to be linearly independent on $E$. Since only the values of the $f_{j}(z)$ on $E$ concern us, we can suppose these functions and $f(z)$ to be polynomials of degree $n-1$. Then the family of polynomials $f(z)-\sum_{1}^{n-1} c_{j} f_{j}(z)$ is the totality of polynomials of degree $n-1$ with precisely one linear relation between their coefficients. If this relation is homogeneous the polynomial zero will belong to the family and $f(z)$ can be exactly represented on $E$; this case is henceforth excluded. In any case not excluded, the relation can be written as $\Gamma[\phi(z)]=1$, where $\phi(z)$ is an arbitrary polynomial of degree $n-1$ and $\Gamma$ is a linear homogeneous function of the coefficients of $\phi(z)$.

With the continued notation $\omega(z)=\Pi\left(z-z_{i}\right)$, we define (complex) weights

$$
w_{i}=\frac{1}{\Gamma\left[\omega_{i}(z)\right]}, \quad \omega_{i}(z) \equiv \omega(z) /\left(z-z_{i}\right),
$$

supposing no denominator to vanish; we discuss later the exceptional case. Consider the polynomials $\phi(z)$ of degree $n-1$ such that

$$
\phi\left(z_{i}\right)=w_{i}\left(z_{i}^{n-1}+d_{1} z_{i}^{n-2}+\cdots+d_{n-1}\right),
$$

for arbitrary coefficients $d_{1}, d_{2}, \cdots, d_{n-1}$. These polynomials are again defined by a single linear relation among their coefficients since the rows

$$
\underset{w_{1} z_{1}}{k} \underset{w_{2} z_{2}}{k}, \cdots, w_{n} z_{n}^{k} \quad(k=0,1, \cdots, n-2)
$$

are linearly independent; the latter fact is a consequence of the identical vanishing of every polynomial of degree $n-2$ which vanishes on $E$.

This linear relation is precisely $\Gamma(\phi)=1$. For we have by Lagrange's formula

$$
\begin{aligned}
\phi(z) & \equiv \sum_{1}^{n} \frac{\phi\left(z_{i}\right)}{\omega^{\prime}\left(z_{i}\right)} \omega_{i}(z), \\
\Gamma[\phi(z)] & =\sum_{1}^{n} \frac{\phi\left(z_{i}\right)}{\omega^{\prime}\left(z_{i}\right)} \Gamma\left[\omega_{i}(z)\right]=\sum_{1}^{n} \frac{\phi\left(z_{i}\right)}{\omega^{\prime}\left(z_{i}\right) w_{i}}=1 ;
\end{aligned}
$$

the last equation is again a consequence of Lagrange's formula

$$
z^{n-1}+d_{1} z^{n-2}+\cdots+d_{n-1} \equiv \sum_{1}^{n} \frac{z_{i}^{n-1}+d_{1} z_{i}^{n-2}+\cdots+d_{n-1}}{\omega^{\prime}\left(z_{i}\right)} \omega_{i}(z),
$$


by inspection of the terms of degree $n-1$ in $z$.

The original problem of minimizing (18) is thus reduced to the problem of minimizing $\max [|\phi(z)|, z$ on $E]$, with $\phi(z)$ defined as in (20). The minimizing polynomial $T_{n-1}(z)$ is (by $\left.\$ 1\right)$ unique and is given by (2) with

$$
\lambda_{i}=\frac{1}{\left|w_{i}\right|\left|\omega^{\prime}\left(z_{i}\right)\right| \sum 1 /\left|w_{i}\right|\left|\omega^{\prime}\left(z_{i}\right)\right|},
$$

namely

$$
T_{n-1}(z) \equiv \sum_{1}^{n} \frac{\omega_{i}(z)}{\left|w_{i}\right|\left|\omega_{i}\left(z_{i}\right)\right| \sum 1 /\left|w_{i}\right|\left|\omega^{\prime}\left(z_{i}\right)\right|} \cdot
$$

Moreover we have the equations for every $i$

$$
\arg \frac{\phi\left(z_{i}\right)}{w_{i}}=\arg \left[\omega^{\prime}\left(z_{i}\right)\right] .
$$

Thus we have the values at $z_{i}$ of the minimizing polynomials $T_{n-1}(z)$ and $\phi_{0}(z)$

$$
\begin{aligned}
T_{\dot{n-1}}\left(z_{i}\right) & =\frac{1}{\left|w_{i}\right|\left|\omega^{\prime}\left(z_{i}\right)\right|} \frac{\omega^{\prime}\left(z_{i}\right)}{\sum 1 /\left|w_{i}\right|\left|\omega^{\prime}\left(z_{i}\right)\right|}, \\
\phi_{0}\left(z_{i}\right) & =w_{i} T_{n-1}\left(z_{i}\right) .
\end{aligned}
$$

TheOREM. The minimizing polynomial $\phi_{0}(z)$ is unique and given by

$$
\phi_{0}(z) \equiv \sum_{1}^{n} \frac{w_{i} \omega_{i}(z)}{\left|w_{i} \omega_{i}\left(z_{i}\right)\right|} / \sum 1 /\left|w_{i} \omega^{\prime}\left(z_{i}\right)\right|
$$

The requirement that (19) shall have a meaning, $i=1,2, \cdots, n$, is essential, as we illustrate by an example. Choose $n=2, z_{1}=0$, $z_{2}=1$, hence $\omega_{1}(z) \equiv z-1$. Choose also $\Gamma\left(a_{0} z+a_{1}\right) \equiv a_{0}+a_{1}$, whence $\Gamma\left[\omega_{1}(z)\right]=0$. The functions $\phi(z)$ with $\Gamma[\phi(z)]=1$ are the polynomials $\left(1-a_{1}\right) z+a_{1}$. Their values at $z_{1}$ and $z_{2}$ are $a_{1}$ and 1 respectively. Here there is no unique polynomial $\phi(z)$ with least maximum modulus. The least possible value for the maximum modulus is unity, and is attained for all $\phi(z)$ with $\left|a_{1}\right| \leqq 1$. Note that for all $\phi(z)$ with $\left|a_{1}\right|<1$, the maximum of the modulus is taken on but once. Thus families of this kind behave very differently from the general type of linear families.

A formula for the most general linear functional $\Gamma[\phi(z)]$ of the coefficients of an arbitrary polynomial $\phi(z)$ of degree $n-1$ is readily found as in (21): 


$$
\phi(z) \equiv \sum_{1}^{n} \frac{\phi\left(z_{i}\right)}{\omega^{\prime}\left(z_{i}\right)} \omega_{i}(z), \quad \Gamma[\phi(z)] \equiv \sum_{1}^{n} \frac{\Gamma\left[\omega_{i}(z)\right]}{\omega_{i}\left(z_{i}\right)} \phi\left(z_{i}\right),
$$

which is the desired relation.

The special case treated in $\$ \S 1$ and 2 is that of a linear family $\phi(z)$ defined by the relation

$$
\sum_{1}^{n} \frac{\phi\left(z_{i}\right)}{w_{i} \omega_{i}\left(z_{i}\right)}=1,
$$

where the $w_{i}$ are positive with sum unity. The Chebyshev polynomial $T_{n-1}(z)$ is not necessarily a member of this family, but related to it by the formula

$$
T_{n-1}\left(z_{i}\right)=\phi_{0}\left(z_{i}\right) / w_{i}
$$

where $\phi_{0}(z)$ is the minimizing polynomial.

We turn to a special case of approximation by a linear family of functions. Let $E_{0}:\left(\alpha_{1}, \alpha_{2}, \cdots, \alpha_{m}\right)$ be a set of points not necessarily all distinct, and let the functional values $\beta_{1}, \beta_{2}, \cdots, \beta_{m}$ be assigned, in the sense that at a point $\alpha_{j}$ of multiplicity $m_{j}$ the corresponding functional values assigned shall be interpreted as the values of $\phi\left(\alpha_{j}\right), \phi^{\prime}\left(\alpha_{j}\right), \cdots, \phi^{\left(m_{j}-1\right)}\left(\alpha_{j}\right)$. Let $E$, disjoint from $E_{0}$, be the point set $z_{1}, z_{2}, \cdots, z_{n}$ of distinct points, let $\mu(z)$ be an arbitrary positive weight function defined on $E$, and let $f(z)$ be an arbitrary function defined on $E$. We consider best approximation on $E$ to $f(z)$ by polynomials $p(z)$ of degree $m+n-2$ which take the prescribed values in the points of $E_{0}$.

Let the polynomial $P(z)$ of degree $m-1$ take the values $\beta_{j}$ in the points $\alpha_{j}$; it is well known that $P(z)$ exists and is unique. We set $\omega_{0}(z) \equiv\left(z-\alpha_{1}\right)\left(z-\alpha_{2}\right) \cdots\left(z-\alpha_{m}\right)$. Then any polynomial $p(z)$ with the required properties can be written as

$$
p(z) \equiv P(z)+\omega_{0}(z) q(z),
$$

where $q(z)$ is a suitable polynomial of degree $n-2$. Moreover, if $q(z)$ is an arbitrary polynomial of degree $n-2$, this equation defines a polynomial $p(z)$ of the class prescribed.

The problem of best approximation is to minimize

$$
\begin{aligned}
\max [\mu(z) \mid f(z)- & p(z) \mid, z \text { on } E] \\
& =\max \left[\mu(z)\left|\omega_{0}(z)\right|\left|\frac{f(z)-P(z)}{\omega_{0}(z)}-q(z)\right|, z \text { on } E\right] ;
\end{aligned}
$$

thus our problem has been reduced to the problem of approximating 
on $E$ to the function $[f(z)-P(z)] / \omega_{0}(z)$ by an arbitrary polynomial $q(z)$ of degree $n-2$, with the positive weight function $\mu(z)\left|\omega_{0}(z)\right|$. All results of $\$ \$ 1-9$ can then be applied, even without our special discussion of general linear families of functions.

As a concluding remark we stress the possibility of extensions of our results to approximation by very general nonlinear families. A natural tool for the investigation of nonlinear families of functions is the linear family obtained by differentiating, in the neighborhood of an approximating function, with respect to the parameters of the family. It should turn out, as directly established [5] for certain families of functions of one real variable, that the behavior of the minimizing function on the given set is essentially the same as in the linear case.

\section{REFERENCES}

1. Ch.-J. de la Vallee Poussin, Sur les polynomes d'approximation d une variable complexe, Bulletin de l'Académie Royale de Belgique (classe des Sciences) vol. 3 (1911) pp. 199-211.

2. L. Fejêr, Uber die Lage der Nullstellen von Polynomen, die aus Minimumforderungen gewisser Art entspringen, Math. Ann. vol. 85 (1922) pp. 41-48.

3. M. Fekete, On the structure of extremal polymomials, Proc. Nat. Acad. Sci. U.S.A. vol. 37 (1951) pp. 95-103.

4. M. Fekete and J. L. von Neumann, Über die Lage der Nullstellen gewisser Minimumpolynome, Jber. Deutschen Math. Verein. vol. 31 (1922) pp. 125-138.

5. Th. Motzkin, Approximation by curves of a unisolvent family, Bull. Amer. Math. Soc. vol. 55 (1949) pp. 789-793.

6. J. L. Walsh, On the location of the roots of certain types of polynomials, Trans. Amer. Math. Soc. vol. 24 (1922) pp. 163-180; Theorem IVa.

7. - A certain two-dimensional locus, Amer. Math. Monthly vol. 29 (1922) pp. 112-114.

National Bureau of Standards, Los Angeles,

University of California, Los ANgeles, and HARVARD UNIVERSITY 